\title{
Polarimetric Approaches for Persistent Scatterers Interferometry
}

\author{
Victor D. Navarro-Sanchez, Student Member, IEEE and Juan M. Lopez-Sanchez, Senior Member, IEEE and \\ Laurent Ferro-Famil Member, IEEE
}

\begin{abstract}
In previous works, a general framework to exploit polarimetric diversity to optimize the results of Persistent Scatterers Interferometry (PSI) was presented, but tested only with dual-pol data. In this paper, the performance of these algorithms is assessed using fully polarimetric data, acquired by Radarsat-2 satellite over the urban area of Barcelona (Spain). In addition, two new highly efficient polarimetric optimization methods, named MIPO and JDPO, are introduced and evaluated. Given the variety of dual-pol configurations provided by current polarimetric satellites, such as TerraSAR-X and Radarsat-2, and the upcoming launch of Sentinel-1, ALOS-2 and Radarsat Constellation Mission, a study has been also carried out in order to determine the best performing dual-pol configurations for polarimetric PSI. Subsidence maps of the area of study are computed for single-pol, dual-pol and full-pol data, which show the increase of density of pixels with valid deformation results as more polarimetric information is made available. In particular, for full-pol data we get an increase of up to 2.5 times more pixels for coherence-based PSI techniques (degraded-resolution), and over 4 times more for amplitude-based approaches (fullresolution), in comparison with single-pol data. Both higher density and quality of pixels yield better results in terms of coverage and accuracy.
\end{abstract}

Index Terms-SAR interferometry, polarimetry, persistent scatterers, subsidence.

\section{INTRODUCTION}

Persistent Scatterers Interferometry (PSI) is a popular tool for the retrieval of accurate subsidence maps, due to its wider coverage and lower cost in comparison with traditional approaches based on the deployment of in-situ instrumentation (e.g. GPS sensor networks). PSI techniques analyze stacks of differential interferograms built from a set of Synthetic Aperture Radar (SAR) images, generally acquired by satellite sensors operating in single-pol configuration [1]-[5]. One of the main features of these techniques is that they start selecting for processing only those pixels that are considered as $a$ priori reliable under certain quality criteria. We usually refer to these pixels as Persistent Scatterer Candidates (PSC) for amplitude based criteria, or Coherent Pixel Candidates (CPC) for selection based on interferometric coherence. Then, in

This work has been supported by the Spanish Ministerio de Educación y Competitividad (MINECO) and European Union FEDER funds under project TEC2011-28201-C02-02.

Victor D. Navarro-Sanchez and Juan M. Lopez-Sanchez are with the Signals, Systems and Telecommunication Group (SST), EPS, University of Alicante, P.O.Box 99, E-03080 Alicante, Spain (e-mail: victor.navarro@ieee.org, juanma-lopez@ieee.org).

Laurent Ferro-Famil is with the SAR Polarimetry Holography Interferometry Radargrammetry Team, Institute of Electronics and Telecommunications of Rennes, University of Rennes 1, 35042 Rennes, France (e-mail: laurent.ferro-famil@univ-rennes1.fr). following steps of the data processing these points will be either confirmed as suitable for the method, or discarded if they do not exhibit the expected behavior. The quality and density of PSC or CPC in the scene is a decisive factor for the accuracy of the outcome of the system.

Though traditionally applied to single-pol data, the availability of improved satellite SAR sensors with polarimetric capabilities such as Radarsat-2, TerraSAR-X or ALOS-PALSAR, makes us wonder how this diversity may help us to improve performance of PSI techniques. To this end, in previous works [6][7] we analyzed the potential use of polarimetry in PSI. A polarimetric optimization approach was proposed, which consisted of a search over the available polarimetric space in order to find, for each pixel, the projection channel that optimized the selection criterion, thus maximizing the number of input points for the PSI method. Polarimetric (multichannel) data can be reprojected onto this optimum channel, getting a complex scattering coefficient analogous to single-pol data. Therefore, this technique can be applied as a preprocessing step for a variety of PSI approaches, with no need of further modification.

In the aforementioned works we tested the approach using TerraSAR-X data in a dual-pol $(H H, V V)$ configuration for two of the most commonly used criteria of selection: the amplitude dispersion index $D_{A}$ computed over a set of calibrated SAR images, and the average interferometric coherence $|\bar{\gamma}|$, computed over a set of multi-looked differential interferograms, obtaining in both cases a significant increase in the number of points selected. We also observed that the analysis of the obtained projection vectors helps us to interpret and identify the different scattering mechanisms present in the area of interest.

In addition to the algorithms introduced in [6][7], two new polarimetric PSI approaches are presented in this paper: MIPO (Mean Intensity Polarimetric Optimization) and JDPO (Joint Diagonalization-based Polarimetric Optimization), a modified version of the coherence optimization algorithm proposed in [8] which makes it suitable for our problem. Alternative ways of using polarimetry in conjunction with satellite PSI have been also proposed by other authors in [9] (separation of different scatterers in the same resolution cell) and [10] (identification of building to ground relative deformation).

New generation satellite SAR sensors are able to provide polarimetric data in a wide range of configurations. Unfortunately, many of them are only capable of acquiring dualpol data, as it is the case for the upcoming European Space Agency's Sentinel-1. In this work, a set of full-pol Single Look 
Complex (SLC) Radarsat-2 images has been used to test the behavior of polarimetric PSI approaches with a complete set of configurations as provided by full polarimetry, including all dual-pol combinations and the synthesized compact or hybridpol [11] mode, which will be made available by the Radarsat Constellation Mission. The main objectives of this work are therefore: (1) to determine the best performing polarimetric PSI approach in terms of coverage and quality of the final results, (2) to assess the benefits of using full-pol data over dual-pol and single-pol data, and (3) to find the best dual-pol acquisition mode (if any) to be used when quad-pol data were not available.

A summary of the general formulation and the new proposed approaches are presented in Section II. Section III describes the available dataset and Section IV presents the obtained results, in form of selection tables and deformation velocity maps.

\section{FORMULATION}

\section{A. Polarimetric SAR interferometry}

A general formulation for polarimetric SAR interferometry (also vector interferometry) was introduced in [12]. For each resolution element, a scattering vector (or target vector) $\mathbf{k}$ is obtained as a vectorization of its scattering matrix $\mathbf{S}$ :

$$
\mathbf{k}=\frac{1}{\sqrt{2}}\left[\begin{array}{c}
H H+V V \\
H H-V V \\
2 H V
\end{array}\right]
$$

where $H H$ and $V V$ stand for the horizontal and vertical copolar channels, respectively, $H V$ is the crosspolar channel, and the Pauli basis has been used for vectorization. Notice that we assume $H V=V H$ due to reciprocity.

In order to generate an interferogram, each target vector $\mathbf{k}$ can be projected onto a unitary complex column vector $\boldsymbol{\omega}$, resulting in a scattering coefficient $\mu$ defined as $\mu=\boldsymbol{\omega}^{\dagger} \mathbf{k}$ [12], where ${ }^{\dagger}$ stands for the hermitian or conjugated transpose. The scattering coefficient $\mu$ is a scalar complex value, obtained as a linear combination of the elements of $\mathbf{k}$. As a complex value, it is analogous to what we could get from a single-channel SAR system for a resolution element. Thus, we can make use of all interferometry techniques developed for single-pol data, including PSI, by applying them to $\mu$.

\section{B. Exhaustive Search Polarimetric Optimization (ESPO)}

In [6][7] we proposed a general framework for PSI polarimetric optimization. The objective is to maximize the number of pixels selected as reliable a priori. This first approach, hereafter named as Exhaustive Search Polarimetric Optimization (ESPO), consists in finding, for each pixel, the projection vector $\boldsymbol{\omega}$ that optimizes the parameter considered as quality criterion. For fully polarimetric data, $\boldsymbol{\omega}$ can be parameterized as follows:

$$
\boldsymbol{\omega}=\left[\begin{array}{l}
\cos (\alpha) \\
\sin (\alpha) \cos (\beta) e^{j \delta} \\
\sin (\alpha) \sin (\beta) e^{j \psi}
\end{array}\right], \quad\left\{\begin{array}{l}
0 \leqslant \alpha \leqslant \pi / 2 \\
0 \leqslant \beta \leqslant \pi / 2 \\
-\pi \leqslant \delta<\pi \\
-\pi \leqslant \psi<\pi
\end{array}\right.
$$

so the problem is reduced to finding four real parameters $\alpha$, $\beta, \delta$ and $\psi$ whose range is finite and known, and whose value is related to the geometric and electromagnetic features of the target [13]. In order to avoid introducing a variable phase term that might be misinterpreted as line-of-sight displacement of the scatterers, we constrain optimum $\boldsymbol{\omega}$ obtained for each pixel to be the same along all the stack of images. This contraint is usually refered as Equal Scattering Mechanisms (ESM) [14], and it can be interpreted as selecting the most stable scattering mechanism over time, according to the chosen criterion of selection. ESPO method can be thought of as an extension to the union algorithm proposed in [15], which consisted in selecting, for each pixel, the best choice from a limited set of conventional channels, e.g. $H H$ and $V V$, instead of searching the whole available polarimetric space. Evidently the union algorithm leads to a sub-optimal solution, but at a much lower computational cost.

ESPO approach has been formulated and tested for two of the most commonly used criteria of selection: minimization of the amplitude dispersion index $D_{A}$, computed over singlelook complex data [1], and maximization of the average magnitude of the interferometric coherences $\overline{|\gamma|}$, estimated using a multi-look scheme [3], [4]. In the context of polarimetric interferometry, $\overline{|\gamma|}$ can be expressed as follows:

$$
\overline{|\gamma|}=\frac{1}{K} \sum_{k=1}^{K}\left|\gamma_{k}\right|, \text { with } \gamma_{k}(\boldsymbol{\omega})=\frac{\boldsymbol{\omega}^{\dagger} \boldsymbol{\Omega}_{i j} \boldsymbol{\omega}}{\sqrt{\boldsymbol{\omega}^{\dagger} \mathbf{T}_{i i} \boldsymbol{\omega}} \sqrt{\boldsymbol{\omega}^{\dagger} \mathbf{T}_{j j} \boldsymbol{\omega}}}
$$

where subscript $k$ denotes the $k$-th interferogram obtained by combining images $i$ and $j$. Polarimetric coherency matrices $\mathbf{T}_{i i}, \mathbf{T}_{j j}$ and polarimetric interferometric cross-correlation matrix $\boldsymbol{\Omega}_{i j}$ are defined as in [12]:

$$
\mathbf{T}_{i i}=E\left[\mathbf{k}_{i} \mathbf{k}_{i}^{\dagger}\right], \quad \mathbf{T}_{j j}=E\left[\mathbf{k}_{j} \mathbf{k}_{j}^{\dagger}\right], \quad \boldsymbol{\Omega}_{i j}=E\left[\mathbf{k}_{i} \mathbf{k}_{j}^{\dagger}\right]
$$

where $E[\cdot]$ is the expectation operator. Notice that such expectations cannot be computed in practice, so they will be replaced by their maximum likelihood estimates that are given, under the usual Gaussian distribution assumption, by the empirical mean evaluated using $L$ independent realizations (or looks) of the target vectors.

Points with $\overline{|\gamma|}$ greater than a given threshold will be considered Coherent Pixel Candidates (CPC).

As for $D_{A}$, it can be expressed as [6][7]:

$$
D_{A}=\frac{\sigma_{a}}{\bar{a}}=\frac{1}{\overline{\left|\boldsymbol{\omega}^{\dagger} \mathbf{k}\right|} \sqrt{N-1}} \sqrt{\sum_{i=1}^{N}\left(\left|\boldsymbol{\omega}^{\dagger} \mathbf{k}_{i}\right|-\overline{\left|\boldsymbol{\omega}^{\dagger} \mathbf{k}\right|}\right)^{2}},
$$

where $N$ is the total number of images and the overline indicates empirical mean value. Points with $D_{A}$ under an established threshold will be selected as Persistent Scatterer Candidates (PSC).

Concerning the actual implementation of the search, in the case of dual-pol data, where only two search parameters $(\alpha$, $\psi$ ) are considered, a conventional fine grid search $\left(\right.$ step $\left.\leqslant 5^{\circ}\right)$ is feasible from the computational viewpoint. Instead, when dealing with fully-polarimetric data the search space is significantly increased, and computational cost rises accordingly. To overcome this drawback, a coarse grid approach $\left(5^{\circ}<\right.$ step 
$\leqslant 15^{\circ}$ ) to avoid falling in local minima plus a conjugated gradient descent scheme is adopted in the implementation of ESPO. In order to avoid the inherent complexity of this numerical search, two new approaches are proposed in the next sections.

\section{Joint Diagonalization-based Polarimetric Optimization (JDPO)}

An efficient alternative approach for interferometric coherence optimization that takes into account the ESM constraint was proposed in [8]. Polarimetric information of each image is first whitened by applying the following transformation to the target vector associated with each pixel: $\tilde{\mathbf{k}}_{i}=\mathbf{T}_{i i}{ }^{\frac{1}{2}} \mathbf{k}_{i}$. Then, the whitened interferometric coherence is given by:

$$
\tilde{\gamma}_{k}(\tilde{\boldsymbol{\omega}})=\tilde{\boldsymbol{\omega}}^{\dagger} \tilde{\boldsymbol{\Omega}}_{i j} \tilde{\boldsymbol{\omega}} \quad \text { with } \quad \tilde{\boldsymbol{\Omega}}_{i j}=\mathbf{T}_{i i}^{-\frac{1}{2}} \boldsymbol{\Omega}_{i j} \mathbf{T}_{j j}^{-\frac{1}{2}} .
$$

The whitened correlation matrix $\tilde{\Omega}_{i j}$ can be written using Special Unitary (SU) operators as:

$$
\tilde{\boldsymbol{\Omega}}_{i j}=\mathbf{U D U}^{\dagger} \text { with } \mathbf{U U}^{\dagger} \text { and }|\mathbf{U}|=+1
$$

where $\mathbf{D}$ is, in general, not diagonal, and $\mathbf{U}$ can be expressed, for the full-pol case, as a function of three orthogonal unitary vectors $\mathbf{U}=\left[\mathbf{u}_{1}, \mathbf{u}_{2}, \mathbf{u}_{3}\right]$. From expressions (6) and (7) it follows that the whitened interferometric coherence values for $\tilde{\boldsymbol{\omega}}_{n}=\mathbf{u}_{\mathbf{n}}$ correspond to the diagonal elements of $\mathbf{D}$, that is, $\tilde{\gamma}_{k}\left(\tilde{\boldsymbol{\omega}}_{n}\right)=d_{n n}, n=1 \ldots q$, with $q$ equal to the dimensionality of $\mathbf{k}$ ( $q=2$ for dual-pol data, $q=3$ for fullpol data). Therefore, the proposed approach consists in finding the SU operator $\mathbf{U}_{o p t}=\left[\mathbf{u}_{o p t 1}, \mathbf{u}_{o p t 2}, \mathbf{u}_{o p t 3}\right]$ that simultaneously diagonalizes (i.e. maximizes $\sum_{n=1}^{3}\left|d_{n n}\right|^{2}$ while keeping $\sum_{i, j=1}^{3}\left|d_{i j}\right|^{2}$ constant) the set of $\tilde{\boldsymbol{\Omega}}_{k}$ matrices, obtaining $\mathbf{D}_{k}$. Operator $\mathbf{U}_{o p t}$ can be computed iteratively in a very efficient way by using the extended Jacobi technique for simultaneous diagonalization described in [16], which constructs $\mathbf{U}_{\text {opt }}$ as a product of plane rotations globally applied to every $\tilde{\boldsymbol{\Omega}}_{k}$. However, some considerations on this solution have to be taken into account for our application.

First of all, notice that we do not have a unique optimum projection vector, but a basis of three orthogonal vectors, each one with an associated coherence value. In an ideal case, that is to say, polarimetrically stationary data with a single dominant scattering mechanism along the whole stack of interferograms, one of the obtained projection vectors will give us the maximum average coherence value. However, when dealing with non purely stationary data, we observe that each of the projection vectors $\tilde{\boldsymbol{\omega}}_{n}=\mathbf{u}_{\text {optn }}$ can lead to the highest coherence values for different subsets of interferograms. Consequently, selecting only one of these mechanisms for the whole set, in order to satisfy the ESM constraint, will get us to a sub-optimal solution. To solve this issue, the adopted approach consists in selecting the projection vector $\tilde{\boldsymbol{\omega}}_{\text {opt }}$ whose associated $\left|\tilde{\gamma}_{k}\right|$ is highest for the majority of interferograms. We observed that the average coherence of the pixel is closely related to the proportion of interferograms sharing the same dominant scattering mechanism so, generally, points with a non stationary behavior will be discarded by coherence thresholding.
The second issue to consider has to do with the unwhitening transformation needed to return to the original basis. To this end we arrived to a different expression to the one proposed in [8]. As demonstrated in Appendix A, $\gamma_{k}\left(\boldsymbol{\omega}_{i}, \boldsymbol{\omega}_{j}\right)=\tilde{\gamma}_{k}\left(\tilde{\boldsymbol{\omega}}_{i}, \tilde{\boldsymbol{\omega}}_{j}\right)$ when projection vectors satisfy:

$$
\begin{gathered}
\tilde{\boldsymbol{\omega}}_{i}=\frac{\mathbf{T}_{i i}^{\frac{1}{2}} \boldsymbol{\omega}_{i}}{\sqrt{\boldsymbol{\omega}_{i}^{\dagger} \mathbf{T}_{i i} \boldsymbol{\omega}_{i}}}, \quad \tilde{\boldsymbol{\omega}}_{j}=\frac{\mathbf{T}_{j j}^{\frac{1}{2}} \boldsymbol{\omega}_{j}}{\sqrt{\boldsymbol{\omega}_{j}^{\dagger} \mathbf{T}_{j j} \boldsymbol{\omega}_{j}}} \\
\boldsymbol{\omega}_{i}=\frac{\mathbf{T}_{i i}^{-\frac{1}{2}} \tilde{\boldsymbol{\omega}}_{i}}{\sqrt{\tilde{\boldsymbol{\omega}}_{i}^{\dagger} \mathbf{T}_{i i}^{-1} \tilde{\boldsymbol{\omega}}_{i}}}, \quad \boldsymbol{\omega}_{j}=\frac{\mathbf{T}_{j j}^{-\frac{1}{2}} \tilde{\boldsymbol{\omega}}_{j}}{\sqrt{\tilde{\boldsymbol{\omega}}_{j}^{\dagger} \mathbf{T}_{j j}^{-1} \tilde{\boldsymbol{\omega}}_{j}}} .
\end{gathered}
$$

From these equations it is extracted that:

$$
\text { When } \mathbf{T}_{i i} \neq \mathbf{T}_{j j}\left\{\begin{array}{l}
\boldsymbol{\omega}_{i}=\boldsymbol{\omega}_{j} \Rightarrow \tilde{\boldsymbol{\omega}}_{i} \neq \tilde{\boldsymbol{\omega}}_{j} \\
\tilde{\boldsymbol{\omega}}_{i}=\tilde{\boldsymbol{\omega}}_{j} \Rightarrow \boldsymbol{\omega}_{i} \neq \boldsymbol{\omega}_{j}
\end{array}\right.
$$

Consequently, an Equal Scattering Mechanism (ESM) scheme in the whitened basis will correspond to a Multiple Scattering Mechanism (MSM) scheme in the original basis. However, from the analysis of both real and simulated data we can conclude that, as long as the matrices Tii, Tjj are relatively similar, the following approximation holds:

$$
\gamma_{k}\left(\boldsymbol{\omega}_{\text {opt }}, \boldsymbol{\omega}_{\text {opt }}\right) \approx \tilde{\gamma}_{k}\left(\tilde{\boldsymbol{\omega}}_{\text {opt }}, \tilde{\boldsymbol{\omega}}_{\text {opt }}\right) \approx \gamma_{k}\left(\boldsymbol{\omega}_{\text {opt }}, \boldsymbol{\omega}_{\text {opt }}\right),
$$

which implies that no transformation is needed for the optimum projection vector. It is important to point out that pixels whose associated $T$ matrices differ significantly along the set of images will generally exhibit an average interferometric coherence magnitude below the quality threshold, and therefore they are likely to be discarded. Actually, being able to detect pixels with such an erratic polarimetric behavior is basic for the proper functioning of PSI algorithms. Consequently, (11) will hold for most of the points prone to be selected as CPC, that is, pixels with a stationary polarimetric behavior.

\section{Mean Intensity Polarimetric Optimization (MIPO)}

As already demonstrated in [6][7], the ESPO approach is an effective algorithm to minimize $D_{A}$. Generally, we assume that low values of $D_{A}$ are associated with stable reflectivity values of point-like scatterers (Persistent Scatterers, PS). As point-like scatterers, they are not affected by spatial decorrelation, and $D_{A}$ becomes a good estimator of phase standard deviation [1]. However, ESPO minimizes $D_{A}$ of $P S$ as well as $D_{A}$ of clutter (i.e. pixels without any point-like scatterer), so more non point-like scatterers are prone to pass the selection stage. Consequently, many of the selected PSC will be discarded in subsequent steps because of their high spatial decorrelation. In this paper we propose an alternative method based on finding the projection vector $\boldsymbol{\omega}$ that optimizes the mean intensity (or average power) for each pixel along the stack of images. This way we make sure that our projection vector is associated with the dominant or more powerful scatterer mechanism in the resolution cell. Afterward, we still can make use of a stability criteria, such as $D_{A}$ thresholding, over the intensity-optimized data. In the following, we will refer to this method as MIPO (Mean Intensity Polarimetric Optimization). 
The intensity of projected signal $\mu$ can be expressed as $I=|\mu|^{2}=\mu \cdot \mu^{*}$, where ${ }^{*}$ stands for conjugation. Under the assumption that, along the stack of $N$ images, $\mathbf{k}$ follows a zero mean, circular complex multivariate Gaussian distribution with $q \times q$ coherency matrix $\mathbf{T}$, denoted as $\mathbf{k} \sim \mathcal{N}_{q}^{\mathbb{C}}(0, \mathbf{T})$, the maximum likelihood estimate of the mean intensity $\bar{I}$ for a given pixel can be obtained as:

$$
\bar{I}=E\left[\mu \cdot \mu^{*}\right]=\boldsymbol{\omega}^{\dagger} \widehat{\mathbf{T}} \boldsymbol{\omega}, \quad \text { with } \quad \widehat{\mathbf{T}}=\frac{1}{N} \sum_{i=1}^{N} \mathbf{k}_{i} \cdot \mathbf{k}_{i}^{\dagger}
$$

where $E[\cdot]$ is the expectation operator, and $\widehat{\mathbf{T}}$ is the maximum likelihood estimate of $\mathbf{T}$. This equation is a quadratic form, where $\widehat{\mathbf{T}}$ is Hermitian semidefinite positive and $\boldsymbol{\omega}$ is unitary. Therefore, maximization of $\bar{I}$ for a given pixel can be posed as an eigenproblem, where the maximum possible value is the maximum eigenvalue of $\widehat{\mathbf{T}}$, and the optimum scattering mechanism is given by its corresponding eigenvector.

\section{E. Summary of approaches}

Following, we provide a comprehensive summary of the presented methods:

- ESPO [6][7]: Consists of a search over the available polarimetric space in order to find the channel that optimizes the quality criterion. This method is suitable for both PSC selection (full resolution) and for CPC selection (degraded resolution, because spatial averaging is required for the estimation of interferometric coherence).

- Union [15]: It can be considered a simplified version of ESPO. For each pixel, we select the best choice from a reduced set of available channels. Hence, no reprojection of data is performed. It is suitable for both PSC and CPC selection.

- JDPO: This algorithm is an adaptation of the one presented in [8]. It finds the optimum projection vector by obtaining the SU operator that best diagonalizes the whole set of whitened interferometric coherency matrices associated with the pixel. This method is not suitable for PSC selection, since it requires spatial averaging for the estimation of interferometric coherency matrices.

- MIPO: Finds the polarimetric channel that optimizes mean intensity of the pixel over time. Suitable for fullresolution criteria such as PSC selection, since it does not require spatial averaging.

\section{DATASET}

A set of 31 full-pol SLC images acquired by Radasat-2 from January 2010 to February 2012 over the urban area of Barcelona (Spain) has been used to test the proposed algorithms. Images have been acquired at a revisit time of 24 days using Radarsat-2 beam mode FQ9: nominal incidence angle of $28^{\circ}$ (near range) to $29.8^{\circ}$ (far range). Nominal azimuth and slant-range resolutions are $7.6 \mathrm{~m}$ and $5.2 \mathrm{~m}$, whereas nominal pixel dimensions are $5.1 \mathrm{~m}$ and $4.7 \mathrm{~m}$, respectively. Therefore, the resulting oversampling factors are 1.49 and 1.11 in azimuth and range.
The processing has been applied over a section of the image of $1400 \times 3600$ pixels, where non-urban areas (mainly sea and mountains) have been masked out. A color composite formed by the Pauli average intensities of the whole area is shown in Fig. 1. The different colors in the composite image provide an insight about the information content of polarimetry. The city is mostly located in the center of the image, showing different dominant channels at different parts. Buildings tend to behave as oriented dihedrals (due to double reflections facade-ground), whose polarimetric response depends highly on the orientation angle (OA) [17]. Dihedrals with OA of $45^{\circ}$ have a dominant cross-polar response, whereas $22.5^{\circ}$ rotated dihedrals reflect equally all four polarimetric channels.

From the full-pol Radarsat-2 data, we can generate different dual-pol sets in order to evaluate performance of the proposed methods when full-pol data are not available. Of special interest are the following combinations: $[H H, V V]$ (TerraSAR-X dual-pol mode), $[H H, H V]$ and $[V V, V H]$ (future ESA Sentinel1 polarimetric modes) and hybrid or compact-pol (available for future Radarsat Constellation Mission, RCM), in which circular polarization is transmitted and linear $\mathrm{H}$ and $\mathrm{V}$ are received: $[R H, R V]$, where $R$ stands for right-circular. Transformation operations between full-pol and hybrid pol are described in [13].

\section{Results}

\section{A. Selection based on average coherence}

In this section we compare performance of ESPO and JDPO methods for average coherence optimization, in terms of CPC density, as well as the best combination of channels if full-pol data are not available. As selection criterion, only those points with $|\bar{\gamma}| \geq 0.7$ will be selected as CPC. In order to estimate coherence, a $7 \times 7$ multi-look is applied (maximum likelihood estimator), which correspond to an equivalent number of looks (ENL) of approximately 30 (see oversampling factors in Section III). This ENL is expected to provide coherence estimates with standard deviation below 0.06 for coherence values above 0.7 according to the expressions derived in [18]. In order to both minimize spatial and temporal decorrelation effects and to keep a tractable stack size, a $150 \mathrm{~m}$ limit for the perpendicular baseline and a 365 days limit for the temporal baseline have been established, resulting in the generation of 137 interferograms from the 31 available images.

As a starting reference, Table I shows CPC selection percentages obtained for each linear, Pauli and hybrid channel synthesized from the available full-pol data, when no optimization is applied. We can freely combine these channels to define different dual-pol schemes, which correspond to different target vector structures. Table II summarizes $C P C$ selection results obtained from applying the aforementioned optimization methods to some of the most frequent polarimetric schemes. The column labelled as Union corresponds to the algorithm proposed in [15] (also mentioned in Section II-A), applied to each set of available channels (components of k). For the ESPO implementation, an initial coarse search grid with a step of $15^{\circ}$ have been employed, followed by a conjugated gradient method. 


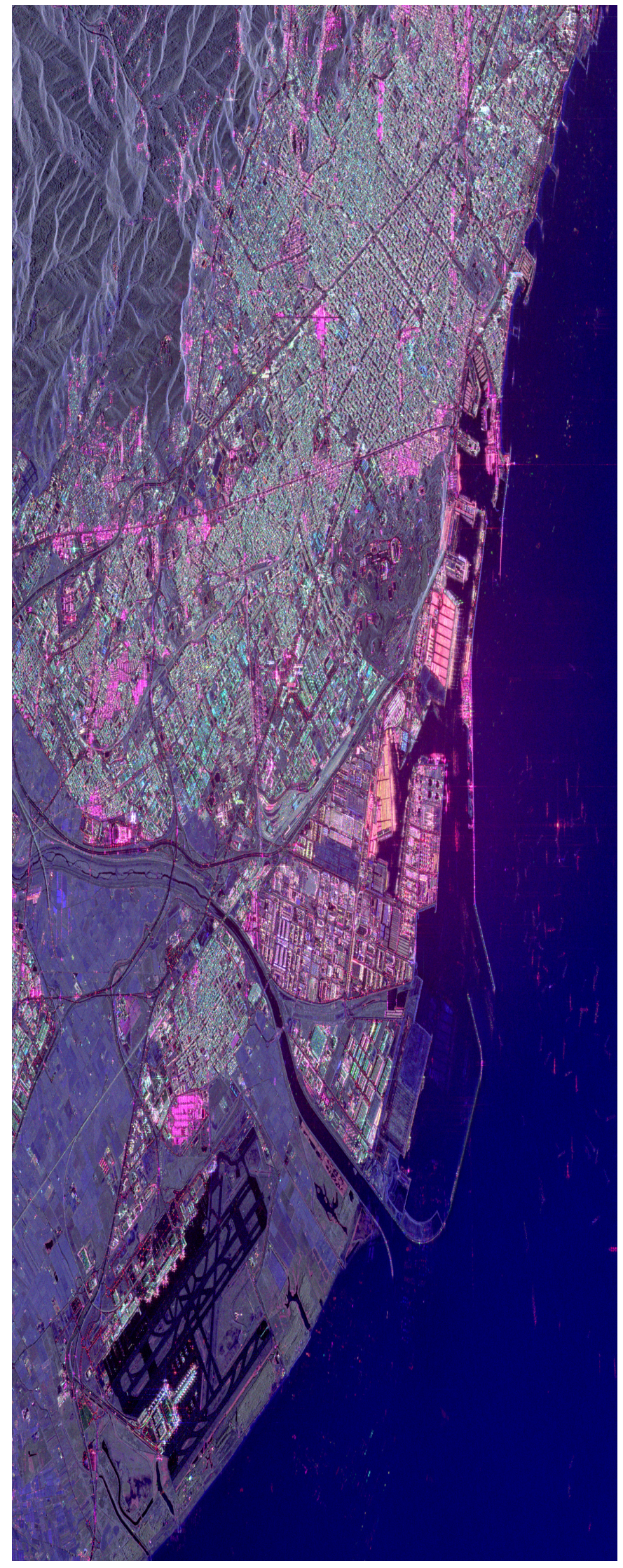

Fig. 1. Composite RGB image of the area under study in Barcelona (Spain) formed by the average intensities. Channels: $\mathrm{R}=\mathrm{HH}-\mathrm{VV}, \mathrm{G}=2 \mathrm{HV}$, $\mathrm{B}=\mathrm{HH}+\mathrm{VV}$
TABLE I

CPC SELECTED FOR DIFFERENT CHANNELS, CONSIDERING A $\overline{|\gamma|}$ THRESHOLD OF 0.7

\begin{tabular}{|c|c||c|c|}
\hline$H H$ & $15.09 \%$ & $R H$ & $15.41 \%$ \\
\hline$V V$ & $14.01 \%$ & $R V$ & $14.53 \%$ \\
\hline$H V$ & $14.69 \%$ & $H H+V V$ & $13.85 \%$ \\
\hline$V H$ & $14.58 \%$ & $H H-V V$ & $16.23 \%$ \\
\hline
\end{tabular}

TABLE II

CPC SELECTED FOR OPTIMIZED DATA CONSIDERING A $\overline{|\gamma|}$ THRESHOLD OF 0.7, FOR DIFFERENT POLARIMETRIC SCHEMES

\begin{tabular}{c|c|c|c|}
$\mathbf{k}$ & Union & ESPO & JDPO \\
\hline$[H H, V V]^{T}$ & $20.08 \%$ & $26.29 \%$ & $25.06 \%$ \\
\hline$[H H, H V]^{T}$ & $21.98 \%$ & $25.82 \%$ & $23.20 \%$ \\
\hline$[V V, V H]^{T}$ & $20.65 \%$ & $25.22 \%$ & $22.80 \%$ \\
\hline$[R H, R V]^{T}$ & $20.43 \%$ & $26.13 \%$ & $25.04 \%$ \\
\hline$\frac{1}{\sqrt{2}}[H H+V V, H H-V V]^{T}$ & $22.10 \%$ & $26.29 \%$ & $25.01 \%$ \\
\hline$\frac{1}{\sqrt{2}}[H H+\sqrt{2} H V, V V]^{T}$ & $24.68 \%$ & $34.65 \%$ & $31.95 \%$ \\
\hline
\end{tabular}

We observe in Table II a significant increase in the number of CPC selected for the three algorithms considered with respect to Table I. Union algorithm performs better when Pauli channels are considered. For a dual-pol configuration $(H H+V V \cup H H-V V)$ it gets an increase of about $46 \%$ more CPC in comparison with $H H$, which is the best single-pol channel. When crosspolar information is added $(H H+V V \cup H H-V V \cup H V)$, the improvement is of about $75 \%$ more CPC selected. In the ESPO case, all the different dual-pol schemes considered lead to similar results, with an important increase of about $75 \%$ more CPC than in singlepol. As for the full-pol configurations, we obtain a dramatic improvement of about $130 \%$ with respect to single-pol. For the JDPO approach, dual-pol $[H H, H V]$ and $[V V, V H]$ (Sentinel-1 polarimetric modes) perform slightly worse than the rest of dual-pol configurations tested, but even so the improvement respect to single $H H$ is significant (about 53\% increase). Other dual-pol schemes considered lead to an improvement of about $66 \%$, whilst both lexicographic and Pauli full-pol setups get near a $90 \%$ increase of density when this algorithm is used.

As expected, the Union algorithm provides a modest improvement in comparison with ESPO and JDPO. ESPO is the one that best maximizes coherence, at the highest computational cost, whilst JDPO appears to be a good trade-off between processing time and increase of average coherence. We can also observe that results improve significantly when full-pol data are available, specially for the ESPO algorithm.

At this stage, selected Coherent Pixel Candidates still have to be confirmed as Coherent Pixels (CP), that is, suitable for the obtainment of valid deformation results. In Section IV-C we present deformation results for those $\mathrm{CP}$ that survive all the steps of the PSI processing chain.

\section{B. Selection based on amplitude dispersion}

Following, we compare ESPO and MIPO techniques for PSC selection optimization. Table III shows selection percentages for conventional channels, and Table IV shows results for the different optimization approaches. In all cases a threshold 
TABLE III

PSC SELECTED FOR DIFFERENT CHANNELS, CONSIDERING A $D_{A}$ THRESHOLD OF 0.3

\begin{tabular}{|c|c||c|c|}
\hline$H H$ & $3.99 \%$ & $R H$ & $4.07 \%$ \\
\hline$V V$ & $3.90 \%$ & $R V$ & $3.97 \%$ \\
\hline$H V$ & $3.80 \%$ & $H H+V V$ & $3.80 \%$ \\
\hline$V H$ & $3.80 \%$ & $H H-V V$ & $4.13 \%$ \\
\hline
\end{tabular}

TABLE IV

PSC SELECTED FOR OPTIMIZED DATA CONSIDERING A $D_{A}$ THRESHOLD OF 0.3 , FOR DIFFERENT POLARIMETRIC SCHEMES

\begin{tabular}{c|c|c|c|}
$\mathbf{k}$ & Union & ESPO & MIPO \\
\hline$[H H, V V]^{T}$ & $6.48 \%$ & $12.83 \%$ & $7.46 \%$ \\
\hline$[H H, H V]^{T}$ & $6.64 \%$ & $12.40 \%$ & $5.87 \%$ \\
\hline$[V V, V H]^{T}$ & $6.59 \%$ & $12.71 \%$ & $6.20 \%$ \\
\hline$[R H, R V]^{T}$ & $6.62 \%$ & $13.05 \%$ & $7.74 \%$ \\
\hline$\frac{1}{\sqrt{2}}[H H+V V, H H-V V]^{T}$ & $6.79 \%$ & $12.83 \%$ & $7.46 \%$ \\
\hline$\frac{1}{\sqrt{2}}[H H+V V, H H-V V, 2 H V]^{T}$ & $8.58 \%$ & $30.75 \%$ & $10.10 \%$ \\
\hline
\end{tabular}

of 0.3 has been employed for the amplitude dispersion index. The increase of PSC in comparison with not optimized data is even more spectacular than in previous cases, since singlelook data are more sensitive to geometrical features that affect polarimetric response, such as orientation and shape of pointlike scatterers.

Looking at the tables, we observe that ESPO clearly outperforms Union and MIPO algorithms in terms of PSC density. For dual-pol sets, Union algorithm reaches a significant increase, of about $70 \%$ compared to $H H$ channel, being the set of Pauli channels the most appropriate choice, as in the CPC case shown in Section IV-A. MIPO algorithm performs slightly better than Union, getting a general increase of about $90 \%$, but for the copolar-crosspolar sets $[H H, H V]$ and $[V V, V H]$, which only exhibit around $50 \%$ increment. Comparatively, ESPO achieves a much more important density improvement, of about $225 \%$ (around 3 times more PSC). For full-pol sets, performance is significantly higher for all methods, though differences between different approaches become more evident. Union algorithm shows an improvement of about $120 \%$, and MIPO gets over $150 \%$ increment of PSC compared to $\mathrm{HH}$. As for the ESPO approach, we observe a dramatic increase of $670 \%$ (almost 8 times more PSC) for both lexicographic and Pauli channel sets. However, as already mentioned in Section II-D, ESPO is characterized by optimizing $D_{A}$ of both actual PS and clutter, so it can be expected that some of the selected points do not correspond to real dominant point-like scatterers. Consequently, they are likely to be discarded in subsequent steps of the process due to high decorrelation rates. On the other hand, it can still help us improving the quality of real PS and revealing hidden or masked ones. Consequently, after the presented PSC filtering stage, the total number of valid PS will be significantly higher than for not optimized data.

\section{Deformation results}

Deformation velocity maps have been generated by following the procedure described in [4] [19]: a network of PSC (for $D_{A}$ criterion, full resolution) or $\mathrm{CPC}$ (for $|\bar{\gamma}|$ criterion, degraded resolution) is constructed (usually by Delaunay triangulation), and a phase model is fitted to each link between points by maximizing its model coherence $\Gamma$, given by:

$$
\Gamma=\frac{1}{K} \sum_{k}^{K}\left|e^{j\left(\Delta \phi_{d i f}^{k}-\Delta \phi_{\text {model }}^{k}\right)}\right|,
$$

where $\Delta \phi_{d i f}^{k}$ stands for the measured interferometric phase difference between connected points, for each interferogram $k$, and $\Delta \phi_{\text {model }}^{k}$ is the interferometric phase difference predicted by the model. In our case, we have used a simple phase model which considers linear deformation velocity $v_{\text {lin }}$ and Digital Elevation Model error $\epsilon_{D E M}$ (both constant for all interferograms):

$$
\phi_{\text {model }}^{k}=\frac{4 \pi B_{\perp}^{k}}{\lambda R \sin (\alpha)}\left(\epsilon_{D E M}^{i}-\epsilon_{D E M}^{j}\right)+\frac{4 \pi}{\lambda} T^{k}\left(v_{\text {lin }}^{i}-v_{\text {lin }}^{j}\right),
$$

where $B_{\perp}^{k}$ and $T^{k}$ are the perpendicular and temporal baselines of interferogram $k$, respectively, $\alpha$ is the observation angle, $R$ is the sensor-target distance and $\lambda$ is the central wavelength of the SAR pulses. Particular details on how to solve this model adjustment problem can be found in [4] [19].

The processing of the PSC/CPC follows a multi-layer scheme [19], that allows higher quality points to be processed first and then used to improve the estimates of subsequent layers of lower quality pixels. For the PSC, three selection layers are considered, with $D_{A}$ thresholds of $0.1,0.2$ and 0.3 respectively. For CPC we considered just two layers, with thresholds of 0.8 and 0.7 . In addition, a threshold for $\Gamma$ can be introduced so that links whose behavior differ significantly from the model are removed. In this work, links below a $\Gamma$ value of 0.8 are discarded. PSC and CPC that become isolated (i.e. not connected to other PSC or CPC via quality links) will not be considered suitable for processing, whilst the rest $\mathrm{O}$ pixel candidates will be confirmed as valid PS or CP. Tables V and VI show a comparison of selection percentages before and after removing isolated pixel candidates, for some channels. Notice that we could avoid discarding some of the candidates by adding redundancy to the pixels network, for example by considering all the possible links between points inside a given radius instead of using Delaunay triangulation, at a cost of a much higher computational cost.

Fig. 2 and Fig. 3 show deformation velocity maps obtained for CP-based and PS-based approaches, respectively. Due to paper extension considerations, only some representative channels are shown: $H H$ (best performing copolar channel), $H V$ (crosspolar channel), MIPO, ESPO and JDPO optimum channels, obtained from both dual-pol and full-pol Pauli channel sets. Percentage of valid CP and PS is shown for comparison with preliminary selection results of CPC and PSC. The increase of the area with deformation information provided by PSI is evident for both criteria considered when more than one channel is used.

Most of the previously selected pixels are confirmed as $\mathrm{CP}$ for the coherence criterion, for both JDPO and ESPO (around $90 \%$ of previously selected CPC). It is also important to point out that, for single-pol data, the ratio between CPC 
TABLE V

COMPARISON OF CP BEFORE AND AFTER DISCARDING ISOLATED CPC, CONSIDERING A $\Gamma$ THRESHOLD OF 0.8 .

\begin{tabular}{c|c|c|}
$\mathbf{k}$ & CP Candidates & Final CP \\
\hline$H H$ & $15.09 \%$ & $13.82 \%$ \\
\hline$H V$ & $14.69 \%$ & $13.45 \%$ \\
\hline JDPO (dual-pol Pauli) & $25.01 \%$ & $22.90 \%$ \\
\hline ESPO (dual-pol Pauli) & $26.29 \%$ & $24.12 \%$ \\
\hline JDPO (full-pol Pauli) & $31.95 \%$ & $29.65 \%$ \\
\hline ESPO (full-pol Pauli) & $34.65 \%$ & $32.12 \%$ \\
\hline
\end{tabular}

and surviving $\mathrm{CP}$ is similar to that of optimized data. As for the amplitude dispersion criterion, we observe that a large amount of points selected for ESPO optimized data are discarded by the posterior processing stages: $77 \%$ of PSC are confirmed as PS for dual-pol, and only $49 \%$ in the case of full-pol data. However, the density of remaining PS still surpasses that obtained by the other techniques, getting an effective increase of about 2.7 times more PS than conventional channels for dual-pol input data, and 4.1 times more density when full-pol data are made available. At a much lower computational cost, MIPO achieves an effective increase of more than twice the number of PS for dual-pol data, and about 2.7 times increment in the case of full-pol, with around 95\% of PSC confirmed as PS.

Deformation maps describe similar features to those shown in [9] presentation slides, obtained from the first 15 images (Jan. - Dec. 2010) of the same Radarsat-2 dataset used in this work. Fig. 4 and Fig. 5 show an augmented view of the deformation velocity maps centered in the area of "El Prat" airport, for both CP and PS approaches. We can observe how optimization reveals new details, such as the southern runway, not present in the HH or HV maps, but shown as stable in the optimized CP maps. Buildings deformation is also described in a much precise way, especially for $D_{A}$ optimized data. We can compare results obtained for this location with those presented in [20] for TerraSAR-X single-pol data. A great degree of correspondence is observed, thought the exact period of study in that reference is not specified.

It is also important to note that the cross-polar channel, $H V$, usually related to random volume scattering over natural surfaces, is also providing reliable deformation maps in this urban area, with a density of $\mathrm{CP}$ comparable to $H H$ and superior in the case of PS. As a matter of fact, in composite RGB image (Fig. 1) a significant cross-polar response (green channel) from urbanized areas is observed. As already mentioned in Section III, this can be explained by the strong cross-polar response from tilted dihedrals (oriented buildings). Therefore, availability of this information is an important factor for the completeness of the area description.

\section{CONCLUSIONS}

In this work, new polarimetric PSI optimization approaches have been presented and evaluated for different dual-pol and full-pol sets synthesized from Radarsat-2 fully polarimetric data. All presented approaches succeed in increasing the density of selected stable pixels, and hence the density of pixels with valid deformation results with respect to singlepol data.
TABLE VI

COMPARISON OF PS BEFORE AND AFTER DISCARDING ISOLATED PSC, CONSIDERING A $\Gamma$ THRESHOLD OF 0.8 .

\begin{tabular}{c|c|c|}
$\mathbf{k}$ & PS Candidates & Final PS \\
\hline$H H$ & $3.99 \%$ & $3.64 \%$ \\
\hline$H V$ & $3.80 \%$ & $3.50 \%$ \\
\hline MIPO (dual-pol Pauli) & $7.46 \%$ & $6.98 \%$ \\
\hline ESPO (dual-pol Pauli) & $12.83 \%$ & $9.85 \%$ \\
\hline MIPO (full-pol Pauli) & $10.10 \%$ & $9.69 \%$ \\
\hline ESPO (full-pol Pauli) & $30.73 \%$ & $15.05 \%$ \\
\hline
\end{tabular}

For techniques based in selection by average coherence (Coherent Pixels, degraded resolution), ESPO approach is the one that provides the largest improvement, with an increase of CP density of around 75\% for dual-pol data, and approximately $130 \%$ for full-pol data, after discarding non valid CPC. Union algorithm and JDPO do also provide a significant improvement at a lower computational cost. As for methods based in amplitude dispersion criterion (Persistent Scatterers, full-resolution), ESPO approach is also the best performing method, with increases of PS density of around 170\% (almost 3 times more PS) for dual-pol sets, and around 310\% (over 4 times more PS) with respect to single-pol, after non valid PSC are discarded.

From the non full-pol sets considered, dual-pol Pauli and hybrid-pol data are the best choices for all of the studied approaches, both CP-based and PS-based. However, notice that in order to generate Pauli channels we need a dual-pol SAR system capable of providing coherent measures of $\mathrm{HH}$ and $V V$. Unlike dual-pol and compact-pol systems, $[H H, V V]$ sensors have the same requirements as full-pol in terms of PRF and reduced swath, since they require multiplexed transmission of horizontal and vertical polarizations. Consequently, hybridpol would be the preferred option when full-pol data are not available.

As future research line, detection and interpretation of multiple stable scattering mechanisms within the same resolution cell will be addressed.

\section{ACKNOWLEDGEMENT}

The Radarsat-2 images were provided by MDA in the framework of the scientific project SOAR-EU 6779. We thank Prof. Jordi Mallorquí (UPC) for providing access to the Radarsat-2 data. Ortophotos have been provided by the Spanish Ministerio de Agricultura, Alimentación y Medio Ambiente (MAAMA).

\section{APPENDIX A}

\section{WHITENING AND UNWHITENING TRANSFORMATIONS}

Relationship between projection vectors in the original and whitened basis is given by equations (8) and (9). Equation (8) 


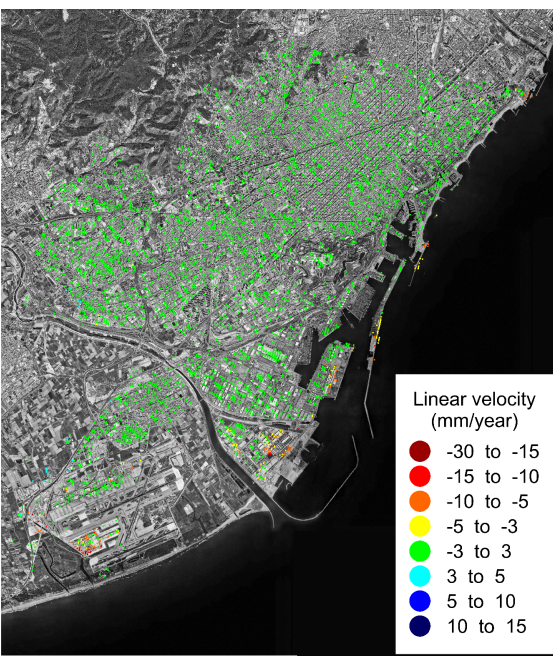

$\mathrm{HH}(\mathrm{CP} 13.82 \%)$

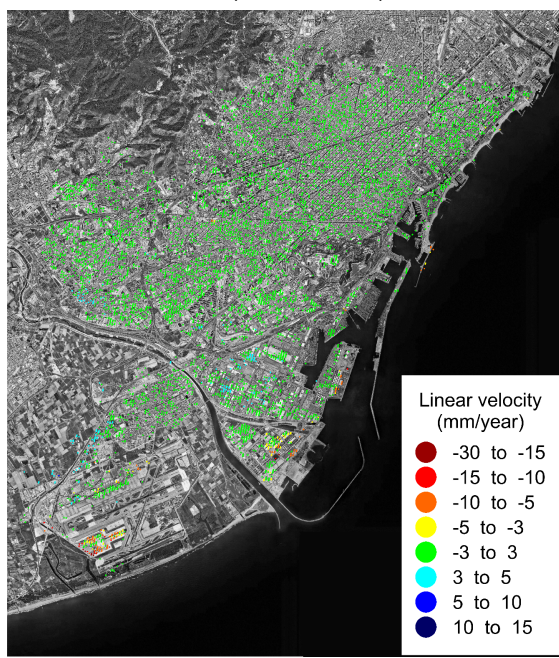

HV (CP 13.45\%)

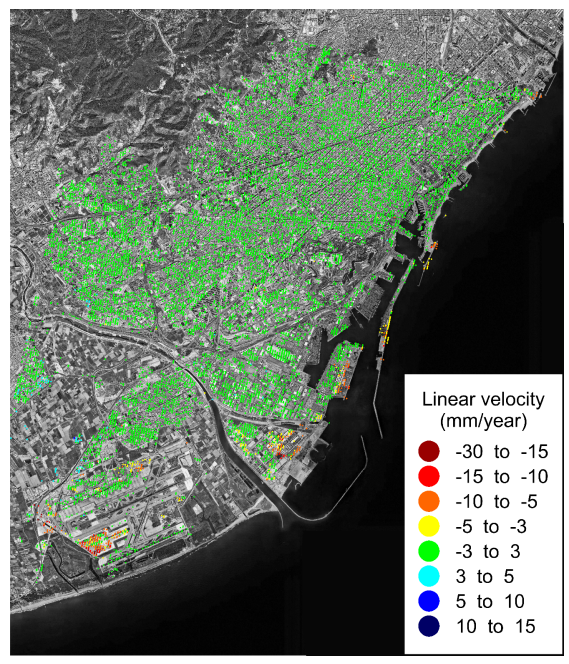

JDPO, Dual-Pol Pauli (CP 22.90\%)

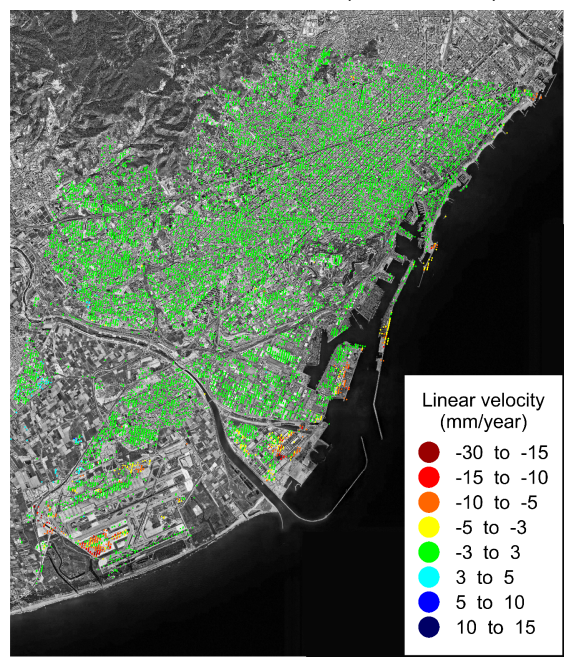

ESPO, Dual-Pol Pauli (CP 24.12\%)

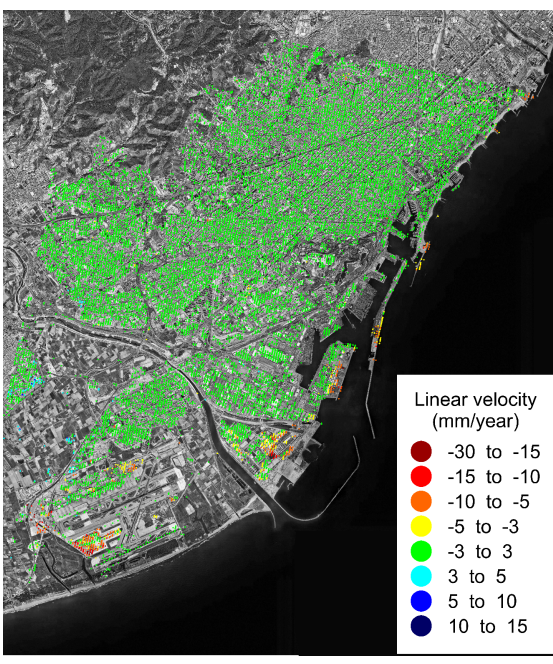

JDPO, Full-Pol Pauli (CP 29.65\%)

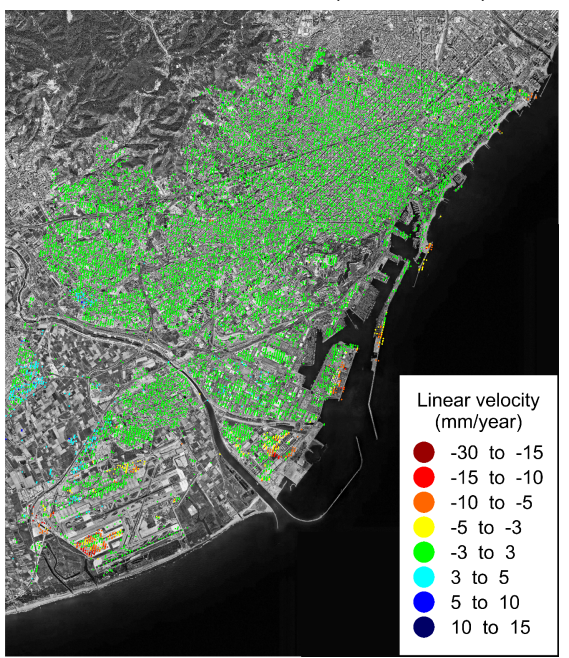

ESPO, Full-Pol Pauli (CP 32.12\%)

Fig. 2. Deformation velocity maps obtained for different polarimetric channels from Coherent Points (degraded resolution, multi-look $7 \times 7$ ).

can be easily demonstrated by substitution into equation (6):

$$
\begin{aligned}
\tilde{\gamma}_{k}\left(\tilde{\boldsymbol{\omega}}_{i}, \tilde{\boldsymbol{\omega}}_{j}\right) & =\tilde{\boldsymbol{\omega}}_{i}^{\dagger} \tilde{\boldsymbol{\Omega}}_{i j} \tilde{\boldsymbol{\omega}}_{j} \\
& =\tilde{\boldsymbol{\omega}}_{i}^{\dagger} \mathbf{T}_{i i}^{-\frac{1}{2}} \boldsymbol{\Omega}_{i j} \mathbf{T}_{j j}^{-\frac{1}{2}} \tilde{\boldsymbol{\omega}}_{j} \\
& =\left(\frac{\mathbf{T}_{i i}^{\frac{1}{2}} \boldsymbol{\omega}_{i}}{\sqrt{\boldsymbol{\omega}_{i}^{\dagger} \mathbf{T}_{i i} \boldsymbol{\omega}_{i}}}\right)^{\dagger} \mathbf{T}_{i i}^{-\frac{1}{2}} \boldsymbol{\Omega}_{i j} \mathbf{T}_{j j}^{-\frac{1}{2}} \frac{\mathbf{T}_{j j}^{\frac{1}{2}} \boldsymbol{\omega}_{j}}{\sqrt{\boldsymbol{\omega}_{j}^{\dagger} \mathbf{T}_{j j} \boldsymbol{\omega}_{j}}} \\
& =\frac{\boldsymbol{\omega}_{i}^{\dagger} \mathbf{T}_{i i}^{\frac{1}{2}} \mathbf{T}_{i i}^{-\frac{1}{2}} \boldsymbol{\Omega}_{i j} \mathbf{T}_{j j}^{-\frac{1}{2}} \mathbf{T}_{j j}^{\frac{1}{2}} \boldsymbol{\omega}_{j}}{\sqrt{\boldsymbol{\omega}_{i}^{\dagger} \mathbf{T}_{i i} \boldsymbol{\omega}_{i}} \sqrt{\boldsymbol{\omega}_{j}^{\dagger} \mathbf{T}_{j j} \boldsymbol{\omega}_{j}}} \\
& =\frac{\boldsymbol{\omega}_{i}^{\dagger} \boldsymbol{\Omega}_{i j} \boldsymbol{\omega}_{j}}{\sqrt{\boldsymbol{\omega}_{i}^{\dagger} \mathbf{T}_{i i} \boldsymbol{\omega}_{i}} \sqrt{\boldsymbol{\omega}_{j}^{\dagger} \mathbf{T}_{j j} \boldsymbol{\omega}_{j}}}=\gamma_{k}\left(\boldsymbol{\omega}_{i}, \boldsymbol{\omega}_{j}\right),(15)
\end{aligned}
$$

and similarly, by substitution of (9) into (3) we obtain:

$$
\begin{aligned}
& \gamma_{k}\left(\boldsymbol{\omega}_{i}, \boldsymbol{\omega}_{j}\right)=\frac{\boldsymbol{\omega}_{i}^{\dagger} \boldsymbol{\Omega}_{i j} \boldsymbol{\omega}_{j}}{\sqrt{\boldsymbol{\omega}_{i}^{\dagger} \mathbf{T}_{i i} \boldsymbol{\omega}_{i}} \sqrt{\boldsymbol{\omega}_{j}^{\dagger} \mathbf{T}_{j j} \boldsymbol{\omega}_{j}}} \\
& =\left(\frac{\mathbf{T}_{i i}^{-\frac{1}{2}} \tilde{\boldsymbol{\omega}}_{i}}{a}\right)^{\dagger} \boldsymbol{\Omega}_{i j} \frac{\mathbf{T}_{j j}^{-\frac{1}{2}} \tilde{\boldsymbol{\omega}}_{j}}{b}
\end{aligned}
$$

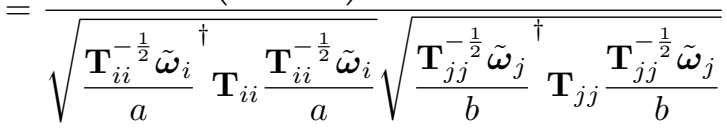

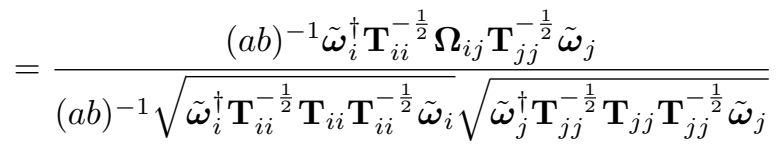

$$
\begin{aligned}
& =\tilde{\boldsymbol{\omega}}_{i}^{\dagger} \mathbf{T}_{i i}^{-\frac{1}{2}} \boldsymbol{\Omega}_{i j} \mathbf{T}_{j j}^{-\frac{1}{2}} \tilde{\boldsymbol{\omega}}_{j}=\tilde{\boldsymbol{\omega}}_{i}^{\dagger} \tilde{\boldsymbol{\Omega}}_{i j} \tilde{\boldsymbol{\omega}}_{j}=\tilde{\gamma}_{k}\left(\tilde{\boldsymbol{\omega}}_{i}, \tilde{\boldsymbol{\omega}}_{j}\right), \\
& \text { with } a=\sqrt{\tilde{\boldsymbol{\omega}}_{i}^{\dagger} \mathbf{T}_{i i}^{-1} \tilde{\boldsymbol{\omega}}_{i}} \text { and } b=\sqrt{\tilde{\boldsymbol{\omega}}_{j}^{\dagger} \mathbf{T}_{j j}^{-1} \tilde{\boldsymbol{\omega}}_{j}}
\end{aligned}
$$

In order to ensure generality of this equations, no ESM constraint has been considered. 

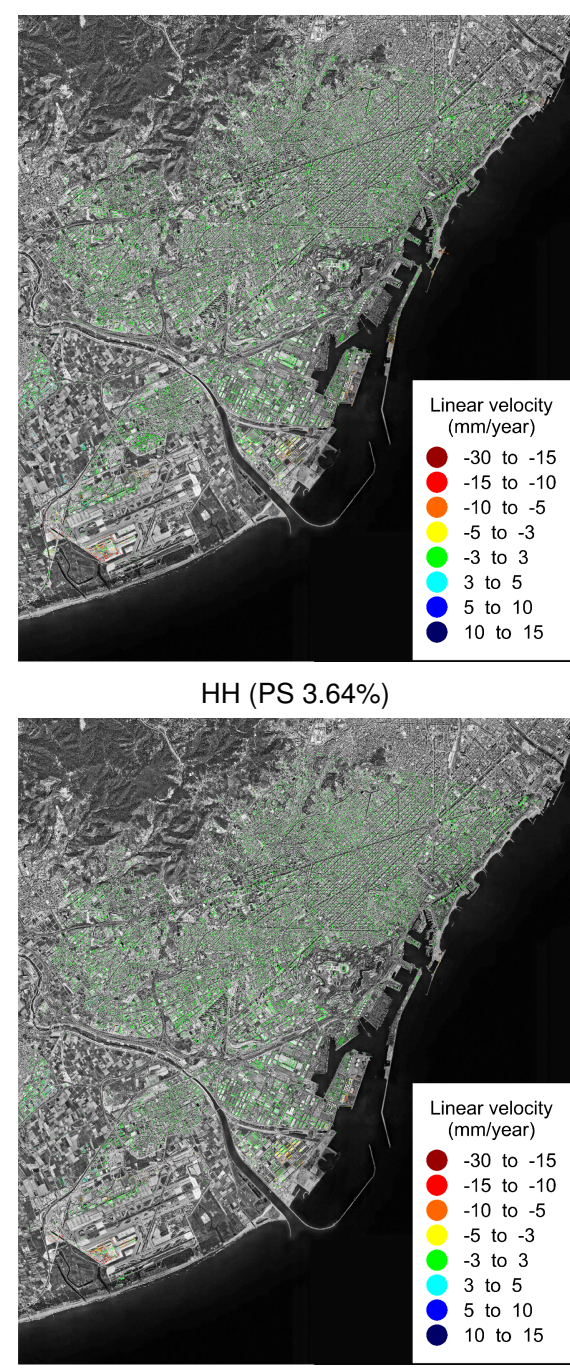

HV (PS 3.50\%)

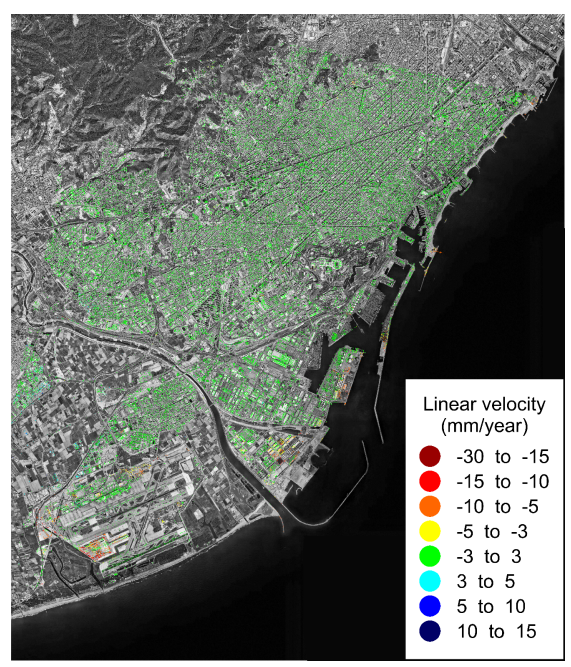

MIPO, Dual-Pol Pauli (PS 6.98\%)

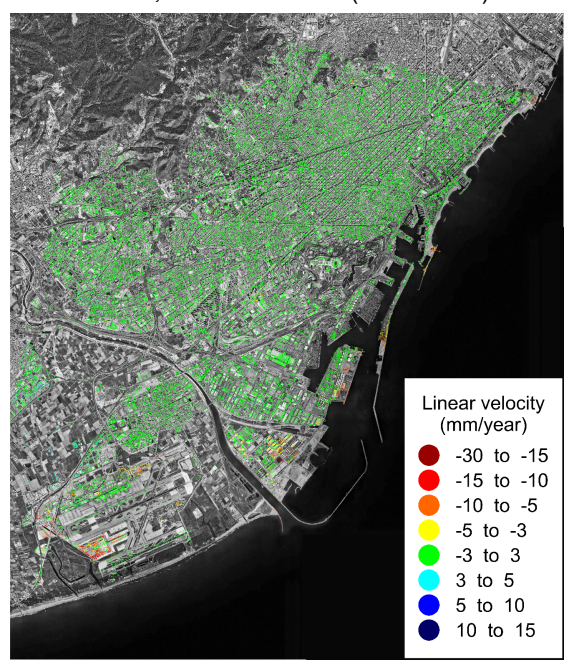

ESPO, Dual-Pol Pauli (PS 9.85\%)

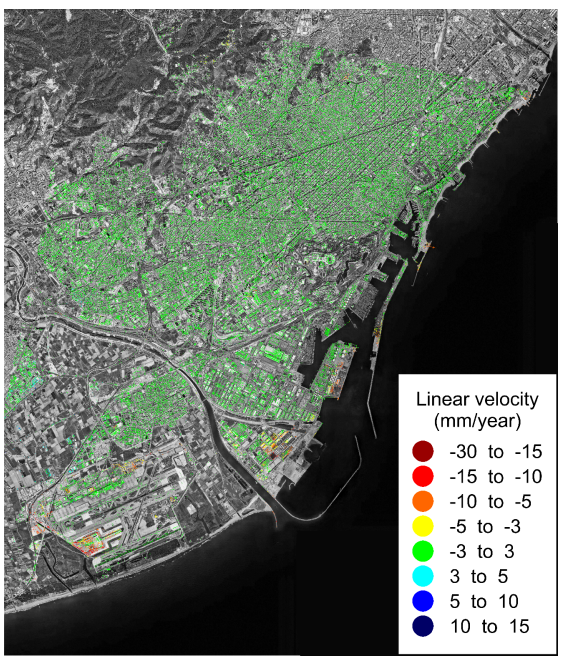

MIPO, Full-Pol Pauli (PS 9.69\%)

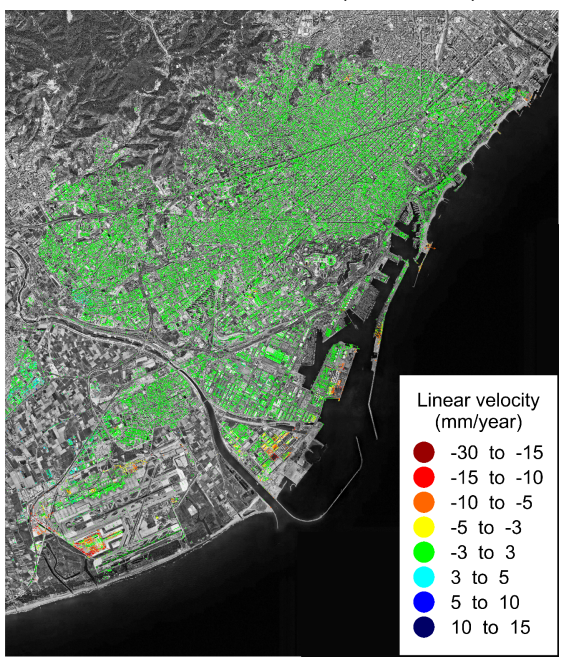

ESPO, Full-Pol Pauli (PS 15.05\%)

Fig. 3. Deformation velocity maps obtained for different polarimetric channels from Persistent Scatterers (full resolution).

\section{REFERENCES}

[1] A. Ferretti, C. Prati, and F. Rocca, "Nonlinear Subsidence Rate Estimation Using Permanent Scatterers in Differential SAR Interferometry," IEEE Trans. Geosci. Remote Sensing, vol. 38, no. 5, pp. 2202 - 2212, Sept. 2000.

[2] - "Permanent scatterers in SAR interferometry," IEEE Trans. Geosci. Remote Sensing, vol. 39, no. 1, pp. 8-20, Jan. 2001.

[3] P. Berardino, G. Fornaro, R. Lanari, and E. Sansosti, "A New Algorithm for Surface Deformation Monitoring Based on Small Baseline Differential SAR Interferograms," IEEE Trans. Geosci. Remote Sensing, vol. 40, no. 11 , pp. 2375-2383, Nov. 2002.

[4] O. Mora, J. J. Mallorquí, and A. Broquetas, "Linear and Nonlinear Terrain Deformation Maps From a Reduced Subset of Interferometric SAR images." IEEE Trans. Geosci. Remote Sensing, vol. 41, no. 10, pp. 2243-2253, Nov. 2003.

[5] A. Ferretti, A. Fumagali, F. Novali, C. Prati, F. Rocca, and A. Rucci, "A New Algorithm for Processing Interferometric Data-Stacks: SqueeSAR," IEEE Trans. Geosci. Remote Sensing, vol. 49, no. 9, pp. 3460-3470, Sept. 2011.

[6] V. D. Navarro-Sanchez, J. M. Lopez-Sanchez, and F. Vicente-Guijalba, "A Contribution of Polarimetry to Satellite Differential SAR Interferometry: Increasing the Number of Pixel Candidates," IEEE Geosci. Remote Sensing Lett., vol. 7, no. 2, pp. 276 -280, Apr. 2010.

[7] V. D. Navarro-Sanchez and J. M. Lopez-Sanchez, "Improvement of Persistent-Scatterer Interferometry Performance by Means of a Polarimetric Optimization," IEEE Geosci. Remote Sensing Lett., vol. 9, no. 4, pp. $609-613$, July 2012.
[8] L. Ferro-Famil, M. Neumann, and Y. Huang, "Multi-baseline Pol-InSAR Statistical Techniques for the Characterization of Distributed Media," in Proceedings of IGARSS'09, Cape Town, South Africa, July 2009.

[9] D. Monells, J. Mallorqui, G. Centolanza, and C. Lopez-Martinez, "Application of Polarimetric Techniques in DInSAR Processing for Space Borne Monitoring," in Proc. of POLinSAR'11, Frascati, Italy, Jan. 2011. [Online]. Available: http://www.polinsar2011.com

[10] P. Dheenathayalan and R. Hanssen, "Target Characterization and Interpretation of Deformation Using Persistent Scatterer Interferometry and Polarimetry," in Proc. of POLinSAR'11, Frascati, Italy, Jan. 2011.

[11] K. Raney, "Hybrid-Polarity SAR Architecture," IEEE Trans. Geosci. Remote Sensing, vol. 45, no. 11, pp. 3397-3404, Nov. 2007.

[12] S. R. Cloude and K. P. Papathanassiou, "Polarimetric SAR Interferometry," IEEE Trans. Geosci. Remote Sensing, vol. 36, no. 5, pp. 1551-1565, 1998.

[13] S. R. Cloude, Polarisation: Applications in Remote Sensing, O. U. Press, Ed. Oxford University Press, 2010.

[14] M. Neumann, L. Ferro-Famil, and A. Reigber, "Multibaseline Polarimetric SAR Interferometry Coherence Optimization," IEEE Geosci. Remote Sensing Lett., vol. 1, no. 5, pp. 93-97, Jan. 2008.

[15] L. Pipia, X. Fabregas, A. Aguasca, C. López-Martínez, S. Duque, J. J. Mallorquí, and J. Marturià, "Polarimetric Differential SAR Interferometry: First Results With Ground-Based Measurements," IEEE Geosci. Remote Sensing Lett., vol. 6, no. 1, pp. 167-171, Jan. 2009.

[16] J. F. Cardoso and A. Souloumiac, "Jacobi angles for simultaneous diagonalization," SIAM J. Mat. Anal. Appl., vol. 17, no. 1, pp. 161164, Jan. 1996

[17] Z. Shan, H. Zhang, C. Wang, W. An, W. An, T. Wu, and X. Chen, "Four- 


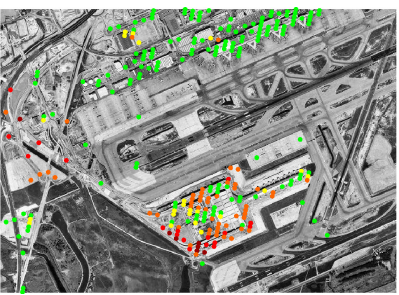

$\mathrm{HH}$

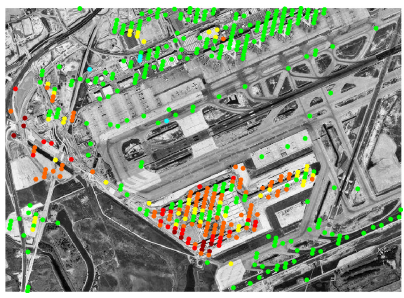

JDPO, Dual-Pol Pauli

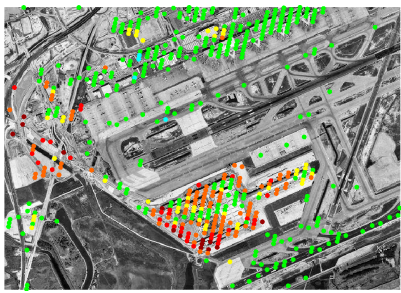

JDPO, Full-Pol Pauli

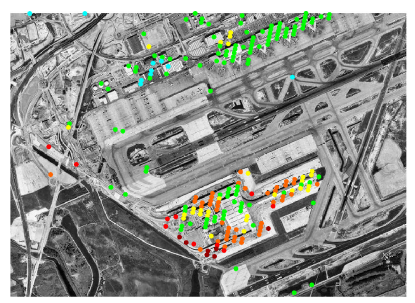

HV

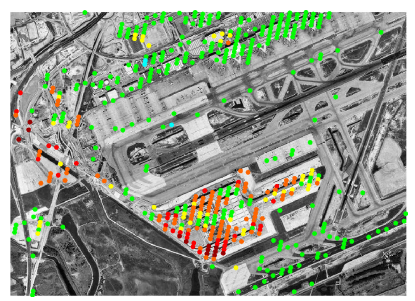

ESPO, Dual-Pol Pauli

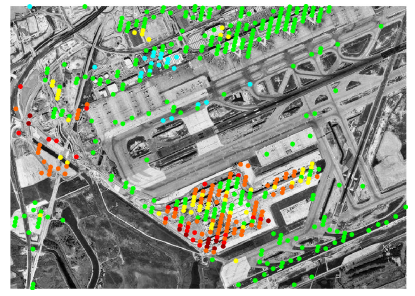

ESPO, Full-Pol Pauli
Fig. 4. Detail of deformation velocity maps (Barcelona - "El Prat"” airport) obtained from Coherent Points. Same colorscale as in Fig. 2 is used.

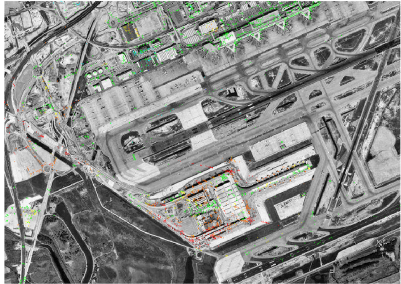

$\mathrm{HH}$

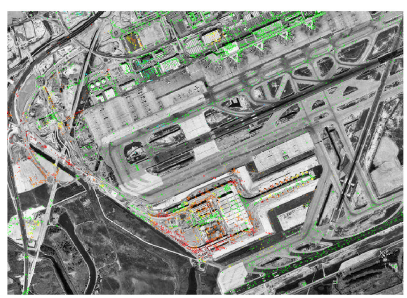

MIPO, Dual-Pol Pauli

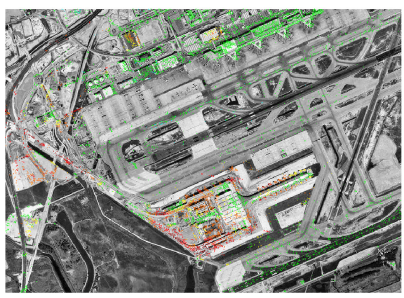

MIPO, Full-Pol Pauli

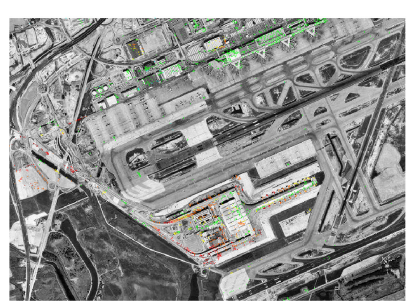

HV

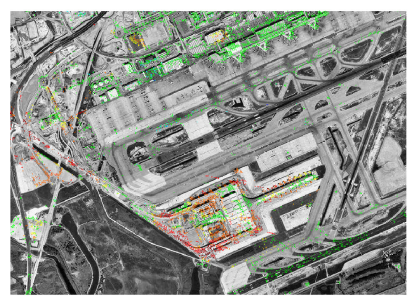

ESPO, Dual-Pol Pauli

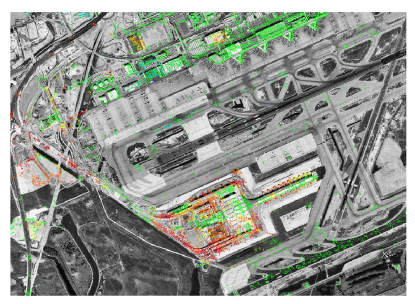

ESPO, Full-Pol Pauli
Fig. 5. Detail of deformation velocity maps (Barcelona - "El Prat"" airport) obtained from Persistent Scatterers. Same colorscale as in Fig. 3 is used.
Component Model-Based Decomposition of Polarimetric SAR Data for Special Ground Objects," IEEE Geosci. Remote Sensing Lett., vol. 9, no. 5, pp. 989-993, Sept. 2012.

[18] R. Touzi, A. Lopes, J. Bruniquel, and W. P. Vachon, "Coherence Estimation for SAR Imagery," IEEE Trans. Geosci. Remote Sensing, vol. 37, no. 1, pp. 135-148, Jan. 1999.

[19] P. Blanco-Sanchez, J. J. Mallorqui, S. Duque, and D. Monells, "The Coherent Pixels Technique (CPT): An Advanced DInSAR Technique for Nonlinear Deformation Monitoring," Pure and Applied Geophysics, vol. 165, pp. 1167-1193, 2008.

[20] O. Monserrat, M. Crossetto, M. Cuevas, and B. Crippa, "The Thermal Expansion Component of Persistent Scatterer Interferometry Observations," IEEE Geosci. Remote Sensing Lett., vol. 8, no. 5, pp. 864-868, Sept. 2011. 